J.H. Mathews

Nagoya Math. J.

Vol. 39 (1970), 149-155

\title{
NORMAL LIGHT INTERIOR FUNCTIONS DEFINED IN THE UNIT DISK
}

\author{
J.H. MATHEWS
}

\section{Preliminaries}

Let $D$ be the unit disk, $C$ the unit circle, and $f$ a continuous function from $D$ into the Riemann sphere $W$. We say that $f$ is normal if $f$ is uniformly continuous with respect to the non-Euclidean hyperbolic metric in $D$ and the chordal metric in $W$. Let $\chi\left(w_{1}, w_{2}\right)$ denote the chordal distance between the points $w_{1}, w_{2} \in W$; and let $\rho\left(z_{1}, z_{2}\right)$ denote the non-Euclidean hyperbolic distance between the points $z_{1}, z_{2} \in \in D$ [6]. If $\left\{z_{n}\right\}$ and $\left\{z_{n}^{\prime}\right\}$ are two sequences of points in $D$ with $\rho\left(z_{n}, z_{n}^{\prime}\right) \rightarrow 0$, we say that $\left\{z_{n}\right\}$ and $\left\{z_{n}^{\prime}\right\}$ are close sequences.

Let $A$ be an open subarc of $C$, possibly $C$ itself. A Koebe sequence of arcs relative to $A$ is a sequence $\left\{J_{n}\right\}$ of Jordan $\operatorname{arcs}$ such that: (a) for every $\varepsilon>0$,

$$
J_{n} \subset\{z \in D:|z-a|<\varepsilon \text { for some } a \in A\}
$$

for all but finitely many $n$, and (b) every open sector $\Delta$ of $D$ subtending an arc of $C$ that lies strictly interior to $A$ has the property that, for all but finitely many $n$, the arc $J_{n}$ contains a subarc $L_{n}$ lying wholly in $\Delta$ except for its two end points which lie on distinct sides of $\Delta$.

We say that the function $f$ has the limit $c$ along the sequence of arcs $\left\{J_{n}\right\}$ (denoted by $f\left(J_{n}\right) \rightarrow c$ ) provided that, for every $\varepsilon>0, \chi\left(c, f\left(J_{n}\right)\right)<\varepsilon$ for all but finitely many $n$.

\section{Factorization of light interior functions}

Let $f$ be a light interior function from $D$ into $W$, i.e. $f$ is an open map which does not take any continum into a single point. Church [4, p. 86] has pointed out that $f$ has the representation $f=g \circ h$ where $h$ is a

Received May 19, 1969.

This paper is part of the author's doctoral thesis directed by Professor Peter Lappan at Michigan State University. 
homeomorphism of $D$ onto a Riemann surface $R$ and $g$ is a non-constant meromorphic function defined on $R$. In view of the uniformization theorem [1, p. 181], there exists a conformal mapping $\varphi$ of $R$ onto either the unit disk or the finite complex plane. We will be concerned with the case when the range of $\varphi$ is the unit disk, but remark that similar results hold when the range is the complex plane. Therefore, if $f$ is a light interior function from $D$ into $W$ then $f$ has a factorization $f=g \circ h$ where $h$ is a homeomorphism of $D$ onto $D$ and $g$ is a non-constant meromorphic function in $D$. Conversely, if $h$ is a homeomorphism of $D$ onto $D$ and $g$ is a non-constant meromorphic function in $D$ then the function $f=g \circ h$ is light interior.

Definition 1. Let $h$ be a homeomorphism of $D$ onto $D$. If $h$ is uniformly continuous with respect to the non-Euclidean hyperbolic metric in both its domain and range then we say that $h$ is HUC.

Definition 2. Let $f$ be a light interior function in $D$ with factorization $f=g \circ h$. If $h$ is HUC then $f$ has a type I factorization; otherwise $f$ has a type II factorization.

THEOREM 1. If $f$ is a light interior function in $D$ then $f$ has a unique factorization type.

Proof. Let $f$ have the factorization $f=g \circ h$. Suppose $f$ also has the factorization $f=G \circ H$. Then as pointed out by Church $\left[4\right.$, p. 86] $h \circ H^{-1}$ is a conformal homeomorphism. In view of Pick's theorem [6, Theorem 15. 1.3, p. 239] both $h \circ H^{-1}$ and $h^{-1} \circ H$ are HUC. Since the composition of two uniformly continuous functions is uniformly continuous, it follows that $h$ is $H U C$ if and only if $H$ is HUC; and the proof of the theorem is complete.

\section{Necessary conditions for both $f$ and $g$ normal}

Noshiro [10, p. 154] has divided the class of normal meromorphic functions in $D$ into two categories which are defined as follows: A normal meromorphic function $g$ in $D$ is of the first category if the normal family $\left\{g\left(\frac{a-z}{1-\bar{a} z}\right)\right.$ : $a \in D\}$ admits no constant limit; otherwise $g$ is of the second category.

THEOREM 2. Let $f$ be a normal light interior function with factorization $f=g \circ h$. If $g$ is a normal meromorphic function then $h$ is normal. Furthermore, if $g$ is a normal meromorphic function of the first category then $h$ is HUC. 
Proof. Let $f$ have the factorization $f=g \circ h$. If $h$ is not normal there exists close sequences $\left\{z_{n}\right\}$ and $\left\{z_{n}^{\prime}\right\}$ such that $h\left(z_{n}\right) \rightarrow e^{i \alpha}$ and $h\left(z_{n}^{\prime}\right) \rightarrow e^{i \beta}$ with $0<\beta-\alpha<2 \pi$ [7]. For each integer $n$, let $J_{n}$ be the non-Euclidean geodesic joining $z_{n}$ to $z_{n}^{\prime}$. Then $\left\{h\left(J_{n}\right)\right\}$ is a sequence of Jordan arcs such that for every $\varepsilon>0$,

$$
h\left(J_{n}\right) \subset\{z \in D: 1-\varepsilon<|z|<1\}
$$

for all but finitely many $n$, and the end points of $h\left(J_{n}\right)$ tend to $e^{i \alpha}$ and $e^{i \beta}$. Choosing a subsequence of $\left\{h\left(J_{n}\right)\right\}$ if necessary, we may assume that there exists a Koebe sequence of $\operatorname{arcs}\left\{L_{n}\right\}$ relative to either the open arc $(\alpha, \beta)$ or the open arc $(\beta, \alpha+2 \pi)$ with $L_{n} \subset h\left(J_{n}\right)$, and a constant $c$ such that $f\left(z_{n}\right) \rightarrow c$.

From the normality of $f$ we have $f\left(J_{n}\right) \rightarrow c$, and it follows that $g\left(L_{n}\right) \rightarrow c$. By a theorem of Bagemihl and Seidel [2, Theorem 1, p. 10], $g \equiv c$ in violation of our hypothesis. Therefore $h$ is normal and the proof of the first part is complete.

Now assume that $g$ is a normal meromorphic function of the first category. If $h$ is not HUC there exists close sequences $\left\{z_{n}\right\}$ and $\left\{z_{n}^{\prime}\right\}$ and a $\delta>0$ with $\rho\left(h\left(z_{n}\right), h\left(z_{n}^{\prime}\right)\right) \geq \delta$, and a constant $c$ such that $f\left(z_{n}\right) \rightarrow c$.

Let $S_{n}(z)=\left(h\left(z_{n}\right)-z\right) /\left(1-\overline{h\left(z_{n}\right)} z\right)$ and let $G_{n}(z)=g\left(S_{n}(z)\right)$. Then the normal family $\left\{G_{n}\right\}$ has a subsequence which converges uniformly on each compact subset of $D$ to a meromorphic function $G[8, \mathrm{p} .53]$. Let $J_{n}$ be the non-Euclidean geodesic joining $z_{n}$ to $z_{n}^{\prime}$ and let $L_{n}=h\left(J_{n}\right)$. Then $d\left(L_{n}\right)=d\left(S_{n}^{-1}\left(L_{n}\right)\right) \geq \delta$, where $d(E)$ is the hyperbolic diameter of the set $E \subset D$. From the normality of $f$ we have $f\left(J_{n}\right) \rightarrow c$, so that $g\left(L_{n}\right) \rightarrow c$, and hence $G_{n}\left(S_{n}^{-1}\left(L_{n}\right)\right) \rightarrow c$. For $r(0 \leq r \leq \delta)$ fixed, there exists a point $Z_{n} \in S_{n}^{-1}\left(L_{n}\right)$ such that $\rho\left(0, Z_{n}\right)=r$. Let $Z_{0}$ be a cluster point of the sequence $\left\{Z_{n}\right\}$ on the circle $\{z: \rho(0, z)=r\}$.

Choosing a subsequence of $\left\{G_{n}\right\}$ if necessary, we may assume that $Z_{n} \rightarrow Z_{0}$ and $G_{n}\left(Z_{n}\right) \rightarrow c$. A familiar argument (see e.g. [3, p. 179]) in the theory of continuous convergence shows that $G\left(Z_{0}\right)=c$. Since $r(0 \leq r \leq \delta)$ was arbitrary, 0 is a limit point of values for which $G$ assumes $c$ and hence $G \equiv c$ in violation our hypothesis. Therefore $h$ is HUC and the proof of the theorem is complete. 


\section{Bounded non-normal light interior functions}

Every bounded holomorphic function is normal, but the following result shows that boundedness is not sufficient for a light interior function to be normal.

THEOREM 3. If a homeomorphism $h$ of $D$ onto $D$ is not HUC, then there exists a Blaschke product $B$ in $D$ such that the bounded light interior function $f=B \circ h$ is not normal.

Proof. If $h$ is not HUC there exists close sequences $\left\{z_{n}\right\}$ and $\left\{z_{n}^{\prime}\right\}$ and a $\delta>0$ such that $\rho\left(h\left(z_{n}\right), h\left(z_{n}^{\prime}\right)\right) \geq \delta$. Let $h\left(z_{n}\right)=w_{n}$ and $h\left(z_{n}^{\prime}\right)=w_{n}^{\prime}$. Since $h$ is uniformly continuous on compact subsets we necessarily have that $\left|z_{n}\right| \rightarrow 1,\left|z_{n}^{\prime}\right| \rightarrow 1,\left|w_{n}\right| \rightarrow 1$, and $\left|w_{n}^{\prime}\right| \rightarrow 1$. Hence, choosing a subsequence of $\left\{w_{n}\right\}$ if necessary, we may assume that $\left\{w_{n}\right\}$ is a Blaschke sequence, i.e. $\sum_{n=1}^{\infty}\left(1-\left|w_{n}\right|\right)<\infty$. There exists a Blaschke subsequence $\left\{w_{n_{k}}\right\}$ of $\left\{w_{n}\right\}$ and a corresponding subsequence $\left\{w_{n_{k}}^{\prime}\right\}$ of $\left\{w_{n}^{\prime}\right\}$ for which $\rho\left(R_{k-1}, r_{k}\right) \geq \tanh ^{-1}\left(1-1 / k^{2}\right)$ where $r_{k}=\min \left\{\left|w_{n_{k}}\right|,\left|w_{n_{k}}^{\prime}\right|\right\}$ and $R_{k}=\max \left\{\left|w_{n_{k}}\right|,\left|w_{n_{k}}^{\prime}\right|\right\}$.

It follows easily that

$$
\rho\left(w_{n_{k}}, w_{n_{j}}^{\prime}\right) \geq \begin{cases}\tanh ^{-1}\left(1-1 /(k+1)^{2}\right) & (1 \leq k<j) \\ \tanh ^{-1}\left(1-1 / k^{2}\right) & (1 \leq j<k),\end{cases}
$$

and hence

$$
\left|\frac{w_{n_{k}}-w_{n_{j}}^{\prime}}{1-\overline{w_{n_{k}}} w_{n_{j}}^{\prime}}\right| \geq \begin{cases}1-1 /(k+1)^{2} & (1 \leq k<j) \\ 1-1 / k^{2} & (1 \leq j<k) .\end{cases}
$$

Recall that $\rho\left(w_{n_{k}}, w_{n_{k}}^{\prime}\right) \geq \delta>0(k=1,2, \cdots)$ so that

$$
\left|\frac{w_{n_{k}}-w_{n_{k}}^{\prime}}{1-w_{n_{k}} w_{n_{k}}^{\prime}}\right| \geq \tanh ^{-1} \delta>0(k=1,2, \cdots) \text {. }
$$

Set $\quad B(z)=\prod_{k=1}^{\infty} \frac{\left|w_{n_{k}}\right|\left(w_{n_{k}}-z\right)}{w_{n_{k}}\left(1-\overline{w_{n_{k}}} z\right)}$.

Consider $B\left(w_{n_{j}}^{\prime}\right)$ for $j \geq 1$,

$$
\left|B\left(w_{n_{j}}^{\prime}\right)\right|=\prod_{k=1}^{j-1}\left|\frac{w_{n_{k}}-w_{n_{j}}^{\prime}}{1-\overline{w_{n_{k}}} w_{n_{j}}^{\prime}}\right| \cdot\left|\frac{w_{n_{j}}-w_{n_{j}}^{\prime}}{1-\overline{w_{n_{j}}} w_{n_{j}}^{\prime}}\right| \cdot \prod_{k=j+1}^{\infty}\left|\frac{w_{n_{k}}-w_{n_{j}}^{\prime}}{1-\overline{w_{n_{k}}} w_{n_{j}}^{\prime}}\right|
$$




$$
\begin{aligned}
& \geq\left(\tanh h^{-1} \delta\right) \prod_{k=1}^{j-1}\left(1-1 /(k+1)^{2}\right) \prod_{k=j+1}^{\infty}\left(1-1 / k^{2}\right) \\
& =\left(\tanh h^{-1} \delta\right) \prod_{k=2}^{\infty}\left(1-1 / k^{2}\right)=1 / 2 \tan h^{-1}(\delta)>0 .
\end{aligned}
$$

Let $f=B \circ h$. By assumption $\left\{z_{n_{k}}\right\}$ and $\left\{z_{n_{h}}^{\prime}\right\}$ are necessarily close sequences with

$$
\lim f\left(z_{n_{k}}\right)=\lim B\left(h\left(z_{n_{k}}\right)\right)=\lim B\left(w_{n_{k}}\right)=0
$$

and $\left|f\left(z_{n_{k}}^{\prime}\right)\right|=\left|B\left(h\left(z_{n_{k}}^{\prime}\right)\right)\right|=\left|B\left(w_{n_{k}}^{\prime}\right)\right| \geq 1 / 2 \tanh ^{-1}(\delta)>0 . \quad$ By a theorem of Lappan [7, Theorem 3, p. 156], $f$ is not normal and the proof is complete.

The previous theorem suggests that the normality of $g$ does not insure the normality of $f$. An even stronger statement is the following result.

Theorem 4. There exists a homeomorphism $h$ of $D$ onto $D$ with the property: If $g$ is a normal meromorphic function in $D$, which has two distinct asymptotic limits, then the light interior function $f=g \circ h$ is not normal.

Since a bounded holomorphic function in $D$ is normal and possesses uncountably many distinct radial limits we obtain the following corollary.

Corollary. There exists a homeomorphism $h$ of $D$ onto $D$ with the property: If $g$ is a non-constant bounded holomorphic function in $D$, then the bounded light interior function $f=g \circ h$ is not normal.

Proof of Theorem 4. Let $\left\{R_{n}\right\}$ be a strictly increasing sequence of nonnegative real numbers with $R_{1}=0$ for which $\rho\left(R_{n}, R_{n+1}\right)=1 / n$. Define the mapping $h$ in $D$ by

$$
h(z)=h\left(r e^{i \theta}\right)=r \exp \left(i \theta+2 \pi i\left(r-R_{n}\right) /\left(R_{n+1}-R_{n}\right)\right)
$$

for $R_{n} \leq r<R_{n+1}(n=1,2, \cdots)$. It is easy to verify that $h$ is a homeomorphism of $D$ onto $D$.

Since $g$ has two distinct asymptotic limits, a theorem of Lehto and Virtanen [8, Theorem 2, p. 53] implies that $g$ has two distinct radial limits. Let $\tau_{\alpha}$ and $\tau_{\beta}$ be the radii which terminate at the points $e^{i \alpha}$ and $e^{i \beta}$, respectively, for which $g\left(r e^{i \alpha}\right) \rightarrow a$ and $g\left(r e^{i \beta}\right) \rightarrow b$ with $b \neq a$.

Now the radii of $D$ are mapped onto spirals by $h^{-1}$. Let $h^{-1}\left(\tau_{\alpha}\right) \cap\left[R_{n}, R_{n+1}\right)$ $=z_{n}$ and $h^{-1}\left(\tau_{\beta}\right) \cap\left[R_{n}, R_{n+1}\right)=z_{n}^{\prime}$. Then $\rho\left(z_{n}, z_{n}^{\prime}\right) \leq \rho\left(R_{n}, R_{n+1}\right)=1 / n \quad$ with 
$f\left(z_{n}\right)=g\left(h\left(z_{n}\right)\right) \rightarrow a$ and $f\left(z_{n}^{\prime}\right)=g\left(h\left(z_{n}^{\prime}\right)\right) \rightarrow b$. Hence, by a theorem of Lappan [7], $f$ is not normal and the theorem is proved.

\section{Sufficient conditions for $f$ normal}

We now determine conditions on $h$ and $g$ which insure the normality of $f$. Since the composition of two uniformly continuous functions is uniformly continuous the first result in this direction is obvious.

THEOREM 5. Let $h$ be a homeomorphism of $D$ onto $D$ which is HUC. If $g$ is a non-constant normal meromorphic function, then the light interior function $f=g \circ h$ is normal. Furthermore, if both $h$ and $h^{-1}$ are HUC, then $g$ is normal if and only if $f$ is normal.

Let $f$ be a light interior function in $D$ with factorization $f=g \circ h$ with $h$ a K-quasiconformal homeomorphism of $D$ onto $D$. We show that $f$ is normal if and only if $g$ is normal. This result was proved by Väisälä [11, Theorem 5, p. 20] whose proof is considerably different.

Theorem 6. If $h$ is a K-quasiconformal homeomorphism of $D$ onto $D$, then both $h$ and $h^{-1}$ are HUC.

THEOREM 7. Let $f$ be a light interior function in $D$ with factorization $f=g \circ h$ with $h$ a K-quasiconformal homeomorphism. Then $f$ is normal if and only if $g$ is normal.

Proof of theorem 6. Since $h$ is $K$-quasiconformal, by a theorem of Mori [9] $h^{-1}$ is also $K$-quasiconformal. Hersch and Pfluger [5] have shown that if $h$ is $K$-quasiconformal then $\rho\left(h(z), h\left(z^{\prime}\right)\right) \leq \Psi_{K}\left(\rho\left(z, z^{\prime}\right)\right)$ where $\Psi_{K}$ is continuous and strictly increasing and defined for all $x \geq 0$ with $\Psi_{K}(0)=0$. It follows easily that $h$ is HUC. Similarly $h^{-1}$ is HUC and the theorem is proved.

Proof of theorem 7. From Theorem 6 both $h$ and $h^{-1}$ are HUC. By Theorem 5, $f$ is normal if and only if $g$ is normal and the theorem is proved.

Definition 3. Let $h$ be a homeomorphism of $D$ onto $D$. Define the set $F(h)$ as follows: $e^{i \theta} \in F(h)$ if there exist close sequences $\left\{z_{n}\right\}$ and $\left\{z_{n}^{\prime}\right\}$ and $a$ $\delta>0$ for which $\rho\left(h\left(z_{n}\right), h\left(z_{n}^{\prime}\right)\right) \geq \delta$ and $h\left(z_{n}\right) \rightarrow e^{i \theta}$. 
Theorem 8. Let $h$ be a normal homeomorphism of $D$ onto $D$. If $g$ is a non-constant normal meromorphic function which is continuous on $D \cup F(h)$, then the light interior function $f=g \circ h$ is normal.

Proof. If $f$ is not normal there exist close sequences $\left\{z_{n}\right\}$ and $\left\{z_{n}^{\prime}\right\}$ such that $f\left(z_{n}\right) \rightarrow a$ and $f\left(z_{n}^{\prime}\right) \rightarrow b$ with $b \neq a$ [7]. It follows from the normality of $g$ that $\left\{h\left(z_{n}\right)\right\}$ and $\left\{h\left(z_{n}^{\prime}\right)\right\}$ are not close. Choosing a subsequence of $\left\{z_{n}\right\}$ and a corresponding subsequence of $\left\{z_{n}^{\prime}\right\}$ if necessary, we may assume that $h\left(z_{n}\right) \rightarrow e^{i \theta}$ and $h\left(z_{n}^{\prime}\right) \rightarrow e^{i \theta}$ with $e^{i \theta} \in F(h)$. But $g$ is continuous on $D \cup F(h)$ and hence $b=\lim f\left(z_{n}^{\prime}\right)=\lim g\left(h\left(z_{n}^{\prime}\right)\right)=\lim g\left(h\left(z_{n}\right)\right)=\lim f\left(z_{n}\right)=a$ which is a contradiction. Therefore $f$ is normal and the proof is complete.

\section{REFERENCES}

[1] L. Ahlfors and L. Sario, Riemann Surfaces, Princeton Univ. Press, Princeton, New Jersey, 1965.

[2 ] F. Bagemihl and W. Seidel, Koebe Arcs and Fatou Points of Normal Functions, Comment. Math. Helv. 36 (1961), 9-18.

[ 3 ] C. Carathéodory, Theory of Functions of a Complex Variable, Vol. I, 2nd ed., Chelsea, New York, 1964.

[4] P. Church, Extensions of Stoillow's Theorem, J. London Math. Soc. 37 (1962), 86-89.

[5] J. Hersch and A. Pfluger, Généralisation du lemme de Schwarz et du principe de la mesure harmonique pour les fonctions pseudo-analytiques, C.R. Acad. Sci. Paris 234 (1952), 43-45.

[6] E. Hille, Analytic Function Theory, Vol. II, Ginn, New York, 1962.

[ 7 ] P. Lappan, Some Results on Harmonic Normal Functions, Math. Z. 90 (1965), 155-159.

[8] O. Lehto and K. Virtanen, Boundary Behavior and Normal Meromorphic Functions, Acta Math. 97 (1957), 47-65.

[9] A. Mori, On Quasi-Conformality and Pseudo-Analyticity, Trans. Amer. Math. Soc. 84 (1957), 56-77.

[10] K. Noshiro, Contributions to the Theory of Meromorphic Functions in the Unit-Circle, J. Fac. Sci. Hokkaido Univ. 7 (1939), 149-159.

[11] J. Väisälä, On Normal Quasiconformal Functions, Ann. Acad. Sci. Fenn. Ser. AI, no. 266 (1959), $33 \mathrm{pp}$.

Michigan State University

and

California State College at Fullerton 